\title{
Use of online dietary recalls to assess adherence to dietary guidelines in people living with and beyond cancer: feasibility and process requirements for ASCOT
}

\author{
R. Conway ${ }^{1}$, S. Esser ${ }^{1}$, M. Michalopoulou ${ }^{1}$, V. Ireland ${ }^{1}$, P. Lally ${ }^{1}$, R. Beeken ${ }^{2}$, A. Fisher ${ }^{1}$ and \\ H. Croker ${ }^{1}$ \\ ${ }^{1}$ Research Department of Behavioural Science and Health, University College London, Gower Street, London, WC1E \\ 6BT England, UK and \\ ${ }^{2}$ Leeds Institute of Health Sciences, University of Leeds, Worsley Building, Clarendon Way, Leeds, LS2 9NL England, \\ $U K$
}

The Advancing Survivorship Cancer Outcomes Trial (ASCOT) is a Randomised Controlled Trial (RCT) of a brief lifestyle intervention for people living with and beyond cancer $(\mathrm{LWBC})^{(1)}$. The intervention aims to improve adherence to World Cancer Research Fund (WCRF) guidelines for diet (as well as physical activity and smoking) ${ }^{(2)}$. Adherence to WCRF dietary guidelines were operationalised in relation to daily intake of fruit and vegetables, red meat, processed meat, fibre, fat and free sugar. To assess dietary intake we aimed to collect two 24-hour recalls at four time points: baseline and 3 months, 6 months and 2 years post intervention. Online dietary recalls were the preferred method of collection for convenience and in order to minimise researcher time and costs.

Here we assess firstly, the feasibility of using the myFood $24^{\mathrm{TM}}$ dietary analysis software to collect online dietary recalls from people $\mathrm{LWBC}^{(3)}$ and secondly, the processing required to generate the cancer relevant dietary parameters of interest.

Participants with a previous diagnosis of breast, prostate or colorectal cancer were recruited through 10 National Health Service Trusts in London and Essex ${ }^{(1)}$. Volunteers with an email address were asked to complete online diet recalls and those unable to do this were given the opportunity to complete phone recalls instead.

At baseline, 1059 Participants completed at least one dietary recall. On the whole participants found online submission acceptable $(65 \%)$. Volunteers preferring to complete telephone recalls $(34 \%)$ did so for reasons such as not being technologically minded or being unable to find specific foods on the programme. For dietary fat it was possible to use myFood24 ${ }^{\mathrm{TM}}$ output directly, but for all other measures additional processing was required. The software did not provide information on intake of red meat, processed meat or free sugars, so additional processing steps were developed to facilitate estimation of these. Additional calculations were also performed to estimate intakes of fruit and vegetables in line with Public Health England recommendations. As AOAC fibre content was absent for the majority of foods these were estimated based on NSP values and alternative sources of information.

Using the online format allowed dietary recalls to be collected from a large number of participants but additional processing was required to assess dietary intakes in relation to WCRF guidelines.

1. Beeken RJ, Croker H, Heinrich M et al. (2016) BMJ Open 6, e011646. doi:10.1136/bmjopen-2016-011646

2. World Cancer Research Fund/American Institute for Cancer Research. Continuous Update Project Expert Report (2018) Survivors of breast and other cancers. Available at canceranddietreport.org

3. Ward HA, McLellan H, Udeh-Momoh C et al. (2019) Nutrients 11(7). pii: E1451. doi: 10.3390/nu11071451. 